\title{
Testing of patients and coronavirus disease 2019 (COVID-19) infection before scheduled deliveries
}

\author{
https://doi.org/10.1515/jpm-2020-0444 \\ Received September 18, 2020; accepted September 20, 2020; \\ published online September 30
}

Keywords: COVID-19; pregnancy; SARS-CoV-2; screening before delivery.

To the Editor,

Recently Herraiz et al. [1] found that the percentage of positive tests in asymptomatic women admitted to delivery was only $0.5 \%$ during the post-peak period in Madrid(1). Their results was Concordant with those of Gagliardi et al. [2] but discordant with those found in others cities such as New York [3-5]. Bianco et al. showed that more than $15 \%$ of asymptomatic maternity patients tested positive for Severe acute respiratory syndrome coronavirus 2 (SARS-CoV-2) infection despite having screened negative with the use of a telephone screening tool. Additionally, 58\% of their asymptomatic, screennegative support persons also tested positive for SARS-CoV-2 infection [4]. Sutton et al. [5] screened also 215 pregnant women who were admitted for delivery in two New York hospitals between March 22 and April 4, 2020 and reported that 33/215 (15.3\%) were positive and 29 $(87.9 \%)$ of the 33 patients positive for SARS-CoV-2 at admission had no symptoms of Coronavirus disease 2019 (COVID-19).

In our tertiary university hospital in Belgium, between March 30 and April 15, 2020, we screened 221 pregnant women for symptoms and performed nasopharyngeal swabs to detect SARS-CoV-2 by real-time polymerase chain reaction (RT-PCR) in all of them on admission to the obstetric ward. RT-PCR was negative in 95.5\% (211/221) of the

*Corresponding author: Prof. Jean Cyr Yombi, Department of Internal Medicine and Infectious Diseases, Cliniques Universitaires Saint-Luc, Université Catholique de Louvain, 10, Av Hippocrate, 1200, Brussels, Belgium, E-mail: jean.yombi@uclouvain.be

Julien De Greef and Leila Belkhir, Department of Internal Medicine and Infectious Diseases, Cliniques Universitaires Saint-Luc, Université Catholique de Louvain, Brussels, Belgium. https://orcid.org/00000002-1701-7584 (L. Belkhir)

Pierre Bernard, Department of Obstetrics, Cliniques Universitaires Saint-Luc, Université Catholique de Louvain, Brussels, Belgium

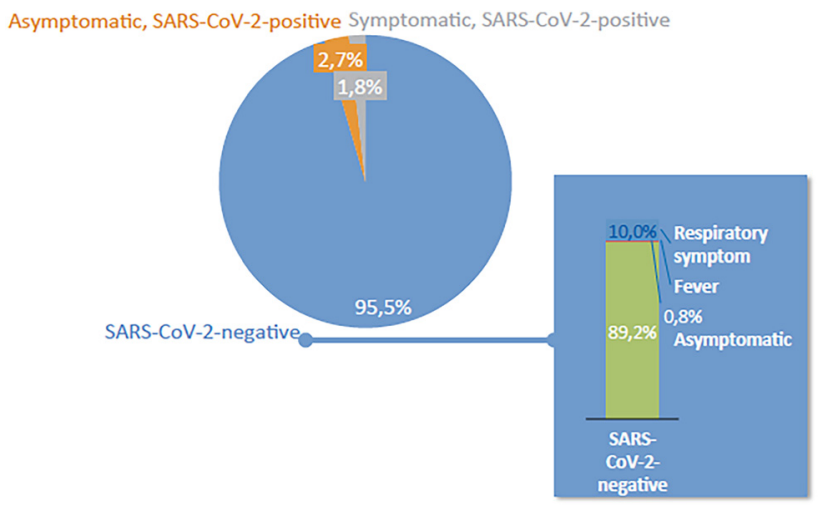

Figure 1: Results of real-time polymerase chain reaction (RT-PCR) on nasopharyngeal swab for 221 pregnant women.

women and positive in 4.5\% (10/221), of whom $4(1.8 \%)$ presented with cough and six (2.7\%) were asymptomatic (Figure 1). Therefore, $60 \%$ of our patients who tested positive did not present any symptoms of COVID-19, concurring with findings reported by Bianco et al. [4] and Sutton et al. [5]. However, The lower proportion of pregnant women testing positive for SARS-CoV-2 in our series (4.5\%) compared to that reported by the pre-cited authors (15.4\%) may be due to variations in the extent of the pandemic in different countries. Indeed our screening was done during period in whom lockdown was adopted in Belgium on April, 1st together with promoting social distancing and hand hygiene. Altogether these data show that the implementation of universal screening of pregnant women presenting for delivery depend of the epidemiology of COVID19 in the city or/and the country.

Research funding: No funding source.

Author contributions: YJC: data collection, conception, wrote the manuscript; JDG, LB, PB: proof reading and correction.

Competing interests: All authors declared no conflict of interest.

Informed consent: All patients agree to be tested at admission and include in this registry.

Ethical approval: Research involving human subjects complied with all relevant national regulations, institutional policies and is in accordance with the tenets of the Helsinki Declaration (as revised in 2013), 
and has been approved by the authors' Institutional Review Board.

\section{References}

1. Herraiz I, Folgueira D, Villalaín C, Forcén L, Delgado R, Galindo A. Universal screening for SARS-CoV-2 before labor admission during Covid-19 pandemic in Madrid. J Perinat Med 2020;48:981-4.

2. Gagliardi L, Danieli R, Suriano G, Vaccaro A, Tripodi G, Rusconi F, et al. Universal SARS-CoV-2 testing of pregnant women admitted for delivery in two Italian regions. Am J Obstet Gynecol 2020 May
12. https://doi.org/10.1016/j.ajog.2020.05.017 [Epub ahead of print].

3. Vintzileos WS, Muscat J, Hoffmann E, Vo D, John NS, Vertichio R, et al. Screening all pregnant women admitted to labor and delivery for the virus responsible for COVID-19. Am J Obstet Gynecol 2020;223:P284-6.

4. Bianco A, Buckley AB, Overbey J, Smilen S, Wagner B, Dinglas C, et al. Testing of patients and support persons for coronavirus disease 2019 (COVID-19) infection before scheduled deliveries. Obstet Gynecol 2020 May 19. https://doi.org/10.1097/AOG. 0000000000003985 [Epub ahead of print].

5. Sutton D, Fuchs K, D'Alton M, Goffman D. Universal screening for SARS-CoV-2 in women admitted for delivery. N Engl J Med $2020 \mathrm{Apr}$ 13. https://doi.org/10.1056/NEJMc2009316 [Epub ahead of print]. 